\title{
Achieving a successful closure of an anterior tympanic membrane perforation. Evidence based systematic review.
}

\author{
Mr. V Visvanathan MSc, FRCS (ORL-HNS), Mr. V Vallamkondu MS, FRCS (ORL-HNS), \\ Miss. A Conybeare MSc, MRCS, DOHNS, Mr. S.K. Bhimrao DM, FRCS (ORL-HNS), \\ University Hospitals of North Midlands NHS Trust
}

\begin{abstract}
Objectives
Surgical repair of a tympanic membrane perforation is a common otological procedure. However achieving a successful closure can be challenging especially if the anterior margin of the tympanic membrane is involved. The aims of this study was to systematically review the literature on evidence published in closure of anterior tympanic membrane perforations.
\end{abstract}

\section{Data extraction}

Two authors independently reviewed titles and abstracts. The PRISMA (preferred reporting items for systematic review and meta-analysis) guidelines were followed.

For the purpose of this study the inclusion criteria were 1) Studies reporting surgical technique for closure of anterior tympanic membrane perforations 2) Primary cases only 3) Articles published in the English language 4) Minimum 6 month follow up 5) Recorded pre and post operative audiometry. The exclusion criteria were 1) Non-English language articles 2) Revision surgery 3 ) No audiometric outcomes .

\section{Results}

On initial search a total of 181 articles were identified (PUBMED 136, COCHRANE 28, EMBASE 17). Using the above criteria 136 articles were excluded. Full text of 45 articles was reviewed and a further 24 excluded. A total of 21 articles fulfilled the criteria for study inclusion.

Our literature search identified several techniques described with appreciable outcomes. Variations in operative technique and audiometric outcomes make it difficult to directly compare/ recommend one technique over another. Although there may be an overlap in described surgical techniques, an attempt has been made to summarise techniques into four categories:

1. Anterior anchoring techniques

2. Lateral graft tympanoplasty

3. Techniques for large/subtotal perforations

4. Endoscopic transcanal technique

\section{Anterior anchoring techniques}

The anterior hitch method described by Kerr is a well-recognized technique for anchoring the anterior margin of the graft. It is useful in perforations with minimal anterior drum remnant. This has a reported success rate of $84.6-94 \%$ with appreciable hearing results.

\section{Lateral Graft Tympanoplasty}

Lateral graft tympanoplasty involves raising a tympanomeatal flap separating the squamous layer from the middle fibrous layer of the tympanic membrane. Vascular strip incisions maintain the blood supply to the entire tympanic membrane, which aids graft uptake.
Angeli $\mathrm{SI}$ et al described the lateral graft tympanoplasty can be undertaken when there is minimal drum anterior to the perforation. In their description the technique can be combined with a mastoidectomy and/or ossiculoplasty as well. In a series of 46 patients, the Modified House Tympanoplasty has a reported $98 \%$ success rate. Anterior canalplasty is often required in the lateral graft technique. Jung TK et al reported a $97 \%$ success rate in a series of 100 patients. They describe a combined mediolateral graft technique. As this technique incorporates the principles of an underlay and overlay technique, it is suitable for all types of anterior perforations.

\section{Techniques for large and subtotal perforation}

Peng and Lalwani reported their technique of 'hammock' tympanoplasty in anterior and large subtotal perforations. The procedure involves creating a wide tympanomeatal flap and placing the graft from the anterior canal wall to the posterior canal wall akin to a hammock. A success rate of $96 \%$ was observed.

In a series of 62 patients, Aidonis et al showed only one failure in their management of total and subtotal perforations. Cartilage is used in an underlay fashion, which is also reinforced by loose areolar tissue. One of the disadvantages of using cartilage especially in mastoid surgery for cholesteatoma is its opaque property making it difficult to assess for recurrent epithelial disease. Diffusion weighted magnetic resonance imaging or second look procedures can be considered in these patients.

\section{Endoscopic Transcanal techniques}

Endoscopic repair of tympanic membrane perforations is gaining popularity. Endoscopic techniques are useful for perforations that are difficult to fully visualize due to an anterior bony overhang but there remains a normal area of tympanic membrane anterior to the perforation.

Endoscopic use minimizes the need for an anterior canalplasty and avoids an external incision. Factors to consider in the use of endoscopes are the learning curve involved in this relatively new technique and single-handed surgery. Success rates between $87 \%$ and $93 \%$ have been reported with its use.

\section{Conclusions}

All published evidence is of level 4 . High quality controlled studies are required to determine the most effective method for closure of anterior tympanic membrane perforation. 